\title{
Challenges and Opportunities in Oncoproteomics
}

Cancer represents one of the major global health concerns affecting the human race today. It affects developing as well as developed nations, and the situation is set to deteriorate worldwide as the global burden of cancer is expected to increase with the continuing growth and ageing of the world's population (Ferlay et al., 2010). To date, there have been substantial advances in basic cancer research which have led to a better understanding of the biological processes underlying disease pathogenesis, a development that has been prompted by the explosive growth in the number of novel highthroughput technologies available for the analysis of genes and their products. We no longer examine the behavior of one single gene or protein but rather scrutinize changes mRNA, miRNA, polymorphisms, and whatnot - at a genomewide level.

The increased basic knowledge about cancer, however, has exposed a level of complexity of the disease that was not obvious a few years ago. For instance, the number of molecular subtypes recognized for given cancer types (e.g. breast, colorectal, leukemia) is increasing rapidly, the daunting heterogeneity of tumour cells both in terms of cell types and differentiation states as well as their interaction with the tumour microenvironment is not yet fully understood, and the ever increasing need for multidisciplinarity in the clinical management of cancer patients and implementation and evaluation of innovations have become more complex. As a result, it is becoming increasingly clear that a significant relief of the cancer burden, in the short-term, may only be achieved by joint collaborative actions directed at improving prevention and therapeutic strategies that successfully increase cure rates. Cancer can be treated more effectively and with less morbidity for patients if malignancies are detected early, i.e. when they are still small and at a localized stage, and early detection is expected to provide a key link between prevention and treatment.

As our understanding of the biology underlying the disease pathogenesis develops, in particular of signaling pathways that are deranged in tumour cells, new biomarkers and targets for drug discovery will be identified and the expectation is that these novel molecular targeted therapies will lead to personalized cancer treatment, i.e. "the right drug for the right patient at the right time". In this context, proteomic technologies are expected to play a key role as they provide invaluable resources to define and characterize regulatory and functional networks and to provide functional insight for developing novel targets for drug development as well as companion diagnostics (Ringborg and Celis, 2010).

The articles in this Thematic issue on "Oncoproteomics" address critical issues and challenges germane to the role of proteomics in the development of more specific targets for drug development and improved biomarkers for diagnosis. This collection of essays is designed to reflect the multiplicity of approaches available to researchers in the field of cancer proteomics and provide the reader with a good and timely overview of the field.

Addressing the need for assessing the state of cell signaling proteins in tumour cells and their normal counterparts when deciding on adequate targeted therapies for individual patients, C. Mueller, L.A. Liotta, and V. Espina (Mueller et al., 2010) describe the profiling of clinical samples with reverse phase protein microarrays (RPMA), a technology that was originally developed to quantitatively measure many proteins simultaneously in extracts from a small number of cells obtained from microdissected tissues from normal and cancer origin. Today, this technology has been widely adopted for preclinical studies using tissues, cells lines, serum, and body fluids and has become a reproducible research clinical trial tool for evaluating the state of cell signaling proteins, predicting therapy response, and prospectively correlating outcome with proteomic expression profiles. The authors present a historical perspective of protein microarrays; describe sample preservation issues and pre-analytical variables associated with proteomic platforms, and report on the goals of current clinical trials in which RPMA are being used. Moreover, they review RPMA literature related to cancer translational research using retrospective analysis and illustrate their applications in pharmaceutical discovery applications.

Cancer phosphoproteomic studies in which abnormal activation of kinase signaling pathways has been shown to be associated with various cancer types are reviewed by H.C. Harsha and A. Pandey (Harsha and Pandey, 2010). They appraise and compare the most commonly used phosphoprotein/peptide enrichment strategies that in combination with quantitative mass spectrometry are currently being used for 
the analysis of the phosphoproteome. Moreover, the authors describe chemical proteomic strategies that have been used to unravel targets of kinase inhibitors which are difficult to characterize using other approaches.

A crucial step towards biomarker and target discovery involves deciding on the source to be used to start a systematic exploration. G.S. Karagiannis, M.P. Pavlou, and E.P. Diamandis (Karagiannis et al., 2010) provide in their review a detailed description of what is currently known about the cancer secretome, i.e. the whole collection of proteins secreted by normal and cancer cells through various secretory pathways. This fluid and sub-fields of secretome analysis such as degradomics, exosomes, and tumour host cell interactions contain many externalised proteins some of which may represent biomarkers or targets for therapeutic intervention. The review addresses challenges and possible clinical applications derived from the proteomic analysis of the secretome, particularly of exosomes.

Early diagnosis of cancer poses a major challenge but it also offers a remarkable possibility and McCarthy and colleagues, (McCarthy et al., 2010) discuss examples of how nanomedicine, in particular targeted nanoagents, can play a role in the diagnosis of cancers. Bioimaging is a rapidly developing field and nanotechnology promises to advance it even further by integrating non-invasive cancer imaging and therapy. This exciting development is partly due to the ability to develop nanoagents that bear multiple functionalities, including those employed for targeting, imaging, and therapy, allowing one to tailor the properties of the nanomaterials. Whereas many nanomaterials exhibit localization to diseased tissues via intrinsic targeting, the addition of targeting ligands, such as antibodies, peptides, aptamers, and small molecules, allows far more sensitive cancer detection.

Antibodies are generally utilized to compare histological traits with protein expression and localization in tissues and K. Schwamborn and R.M. Caprioli (2010) describe how Imaging Mass Spectrometry, a relatively new technology that allows the simultaneous measurement of multiple analytes (proteins, peptides, drugs, lipids) in intact tissue sections, has played a role in cancer research. In particular, the analysis of proteins has proven of value in the discovery setting, and has led to the identification of novel markers for both prognosis and response to treatment. Validation studies are seeing as key for establishing the technology as a key resource in the translational continuum.

Finally, Moreira and colleagues (Moreira et al., 2010) describe the strategies underlying their systematic proteomic studies aimed at characterizing the phenotypes of the different cell subpopulations present in normal human mammary tissue. The ultimate goal is to identify biomarkers that can better determine the outcome of early breast lesions by identifying subpopulations of cells in breast premalignant disease that are at high-risk of progression to invasive disease. Using data generated by 2D gel-based proteomic profiling, these authors compiled a protein database of normal human breast epithelial tissue, and identified biomarkers that characterize the molecular phenotypes of the various cell types and precursors - generated by the stem cell hierarchy - that are present in normal and benign conditions of the breast.

We are indebted to all the authors for providing an excellent and timely overview of their respective fields of expertise and to the staff at Elsevier for professional help.

\section{R E F E R E N C E S}

Ferlay, J., Shin, H.R., Bray, F., Forman, D., Mathers, C., Parkin, D.M., 2010. Estimates of worldwide burden of cancer in 2008: GLOBOCAN 2008. Int. J. Cancer. doi:10.1002/ijc.25516.

Harsha, H.C., Pandey, A., 2010. Phosphoproteomics in cancer. Mol Oncol. 4 (6), 482-495.

Karagiannis, G.S., Pavlou, M.P., Diamandis, E.P., 2010. Cancer secretomics reveal pathophysiological pathways in cancer molecular oncology. Mol Oncol. 4 (6), 496-510.

Mueller, C., Liotta, L.A., Espina, V., 2010. Reverse phase protein microarrays advance to use in clinical trials. Mol Oncol. 4 (6), 461-481.

McCarthy, J.R., Bhaumik, J., Karver, M.R., Erdem, S.S., Weissleder, R., 2010. Targeted nanoagents for the detection of cancers. Mol Oncol. 4 (6), 511-528.

Moreira, J.M.A., Cabezon, T., Gromova, I., Gromov, P., Timmermans-Wielenga, V., Machado, I., Llombart-Bosch, A., Kroman, N., Rank, F., Celis, J.E., 2010. Tissue proteomics of the human mammary gland: Towards an abridged definition of the molecular phenotypes underlying epithelial normalcy. Mol Oncol. 4 (6), 539-561.

Ringborg, U., Celis, J.E., 2010. United we stand. Public Service Review: European Union 20, 199-200.

Schwamborn, K., Caprioli, R.M., 2010. MALDI imaging mass spectrometry - Painting molecular pictures. Mol Oncol. 4 (6), 529-538.

Julio E. Celis* José M.A. Moreira Issue editors

Dept. of Proteomics in Cancer, Institute of Cancer Biology, Danish Cancer Society, Copenhagen, Denmark

${ }^{*}$ Corresponding author.

E-mail address: molonc@cancer.dk (J. E. Celis)

1574-7891/\$ - see front matter (C) 2010 Federation of European Biochemical Societies. Published by Elsevier B.V. All rights reserved. doi:10.1016/j.molonc.2010.10.001 